\title{
Integration of Brain Signals in Multimodal Bedside Monitoring After Traumatic Brain Injury
}

\author{
Emmanuel Carrera*, ${ }^{*}$, Luzius A Steiner ${ }^{2}$, Ken Brady ${ }^{3}$, Christian Zweifel $^{1}$, Gianluca Castellani ${ }^{1}$, \\ Magdalena Hiler ${ }^{1}$, Piotr Smielewski ${ }^{1}$ and Marek Czosnyka ${ }^{1}$
}

\author{
${ }^{l}$ Neurosurgery Unit, Department of Clinical Neurosciences, Cambridge University, United Kingdom; ${ }^{2}$ Department of \\ Anesthesiology, University Hospital Lausanne, Lausanne, Switzerland; ${ }^{3}$ Department of Pediatric Neurointensive care, \\ Johns Hopkins Hospital, Baltimore, USA
}

\begin{abstract}
Background: During the last decade, with the development of on-line monitoring and waveform analysis, integration of brain signals has increasingly been used in patients with traumatic brain injury (TBI) to guide clinical management and predict outcome.

Objective: The goal of this review is to describe current methodology for brain signal integration in TBI patients focusing on 'reactivity indices'.

Methods: We reviewed selected techniques to monitor patients in the acute phase of TBI using the comparison and integration of different physiological signals. The autoregulation indices, Mx and Mxa were defined as the moving correlation between cerebral blood flow velocities (CBFV) and cerebral perfusion pressure (CPP) or arterial blood pressure (ABP), respectively. The cerebrovascular pressure reactivity index $\mathrm{PRx}$ was defined as the moving correlation coefficient between $\mathrm{ABP}$ and intracranial pressure (ICP). The oxygen reactivity index, Orx, was defined as the moving correlation coefficient between brain tissue oxygenation and CPP. Finally, Tox was defined as the moving correlation coefficient between brain tissue oxygenation measured by Near Infrared Spectroscopy (NIRS) and ABP.

Results: Both Mx >0.3 and Mxa >0.45 as well as an asymmetry of Mx between both hemispheres of the brain in the acute phase were associated with poor outcome. PRx may be used to determine the individual optimal CPP and is also a powerful and independent predictor of outcome. Orx showed conflicting results and more studies are need to determine its role in the acute setting of TBI. Studies concerning the role of NIRS in the acute phase of TBI are ongoing.

Conclusion: Multimodal neuromonitoring (MNM) is useful in the neuroICU as it provides valuable insights into the pathophysiological mechanisms occurring in the acute phase of TBI. Furthermore, indices obtained with MNM have been shown to be strong predictors of outcome. Further studies combining current and emergent techniques such as NIRS emphasize on the ability for MNM to continuously evolve.
\end{abstract}

Keywords: Traumatic brain injury, cerebral autoregulation, monitoring, intracranial pressure, cerebral blood flow, transcranial doppler, cerebral perfusion pressure.

\section{A. INTRODUCTION}

With the development of dedicated neurocritical care, different strategies have emerged in the management of patients suffering from severe traumatic brain injury (TBI) [1]. Today, brain monitoring plays a key role in the management of these patients who benefit, as a standard of care, from continuous monitoring of intracranial pressure (ICP), arterial blood pressure (ABP) and heart rate. These parameters may be supplemented, depending on the patient status and equipment availability, with adjunctive monitoring modalities including transcranial Doppler (TCD), brain tissue oxygenation $\left(\mathrm{PbtO}_{2}\right)$, microdialysis, and perhaps near infrared spectroscopy (NIRS) [2,3].

ICP monitoring in TBI patients is not a recent development. The first reports of ICP monitoring were made in 1951

*Address correspondence to this author at the Academic Neurosurgical Unit, Department of Clinical Neurosciences, Level 4, A Block, Addenbrooke's Hospital, Cambridge CB2 2QQ, UK; Tel: + 441223331 763; Fax: + 441223216 326; E-mail: emmanuelcarrera@yahoo.com by Guillaume and Janny [4]. Since then, major advances have been made in the field of brain monitoring, especially during the last decade, with increasing performance in data recording, waveform analysis, and data storage. Computerassisted analysis of primary waveforms from clinical monitors can describe subtle waveform properties including simple metrics such as mean, minimum or maximum values, and more complex analyses of waveform shape. With currently available software [5,6], such analyses may be performed at the bedside in real time at a single time point or continuously. Describing relationships between diverse monitors in the neuro ICU has been particularly useful and has lead to the development of multiple indices, showing physiologic properties not apparent in the isolated monitors. Many of these metrics, including the indices of autoregulation discussed in this manuscript are associated with outcome following TBI. More importantly, they can be used at the bedside to guide clinical decision making. The study of these indices is at the basis of the concept of "multimodality monitoring" of brain function $[2,3]$. 
a

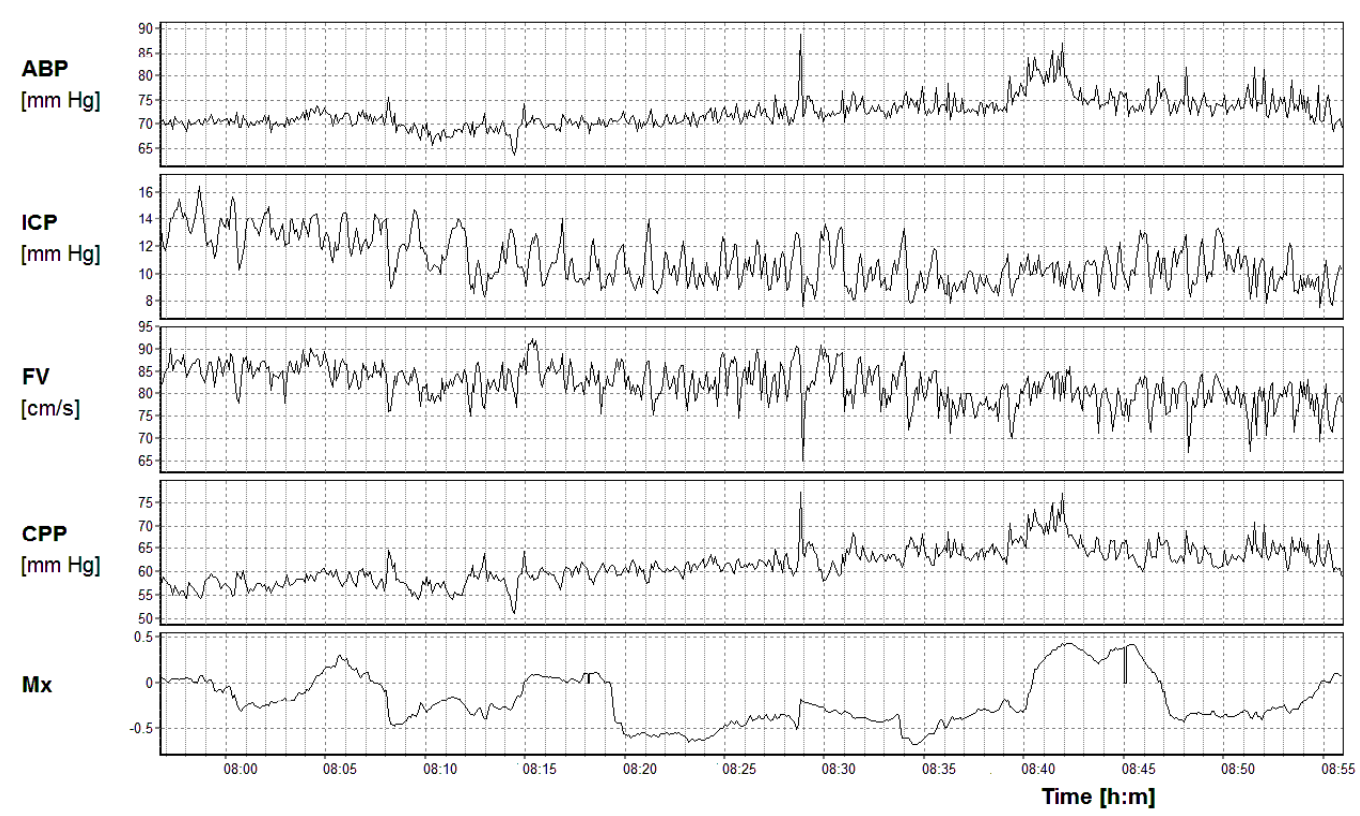

b

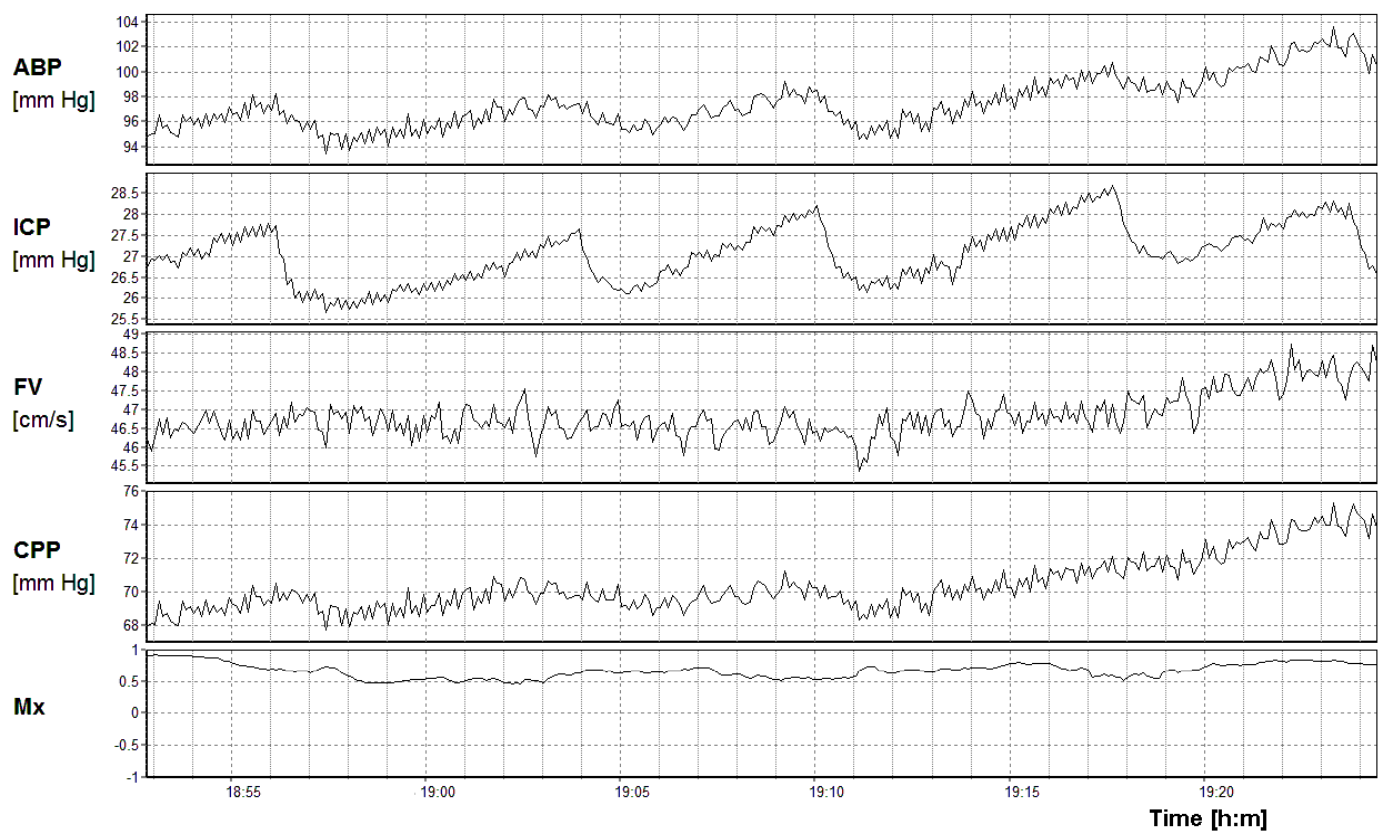

Fig. (1). Examples of monitoring of the autoregulation index Mx in a patient after TBI with a) good outcome - mean ICP was normal. Mx was on average negative (-0.2) with only a short elevation above 0.3 associated with unstable arterial blood pressure b) unfavorable outcome. ICP was elevated and showed a regular pattern of changes, although of small magnitude. Blood flow velocity was obviously correlated with increasing CPP. Mx was continuously positive, suggesting autoregulatory failure.

ICP- intracranial pressure, ABP- arterial blood pressure, CPP- cerebral perfusion pressure, FV- blood flow velocity , Mx- autoregulation index.

In this review, we will first discuss the current evidence related to multimodality monitoring, focusing on monitoring of ICP, cerebral blood flow velocity (CBFV) and ABP. In a second part, we will present the new developments and perspectives in the field of cerebral multimodality monitoring.

\section{B. TRANSCRANIAL DOPPLER AND CEREBRAL BLOOD FLOW VELOCTIES}

Cerebral autoregulation acts as a protective mechanism to maintain cerebral blood flow (CBF) relatively stable despite fluctuations in cerebral perfusion pressure (CPP). Using 


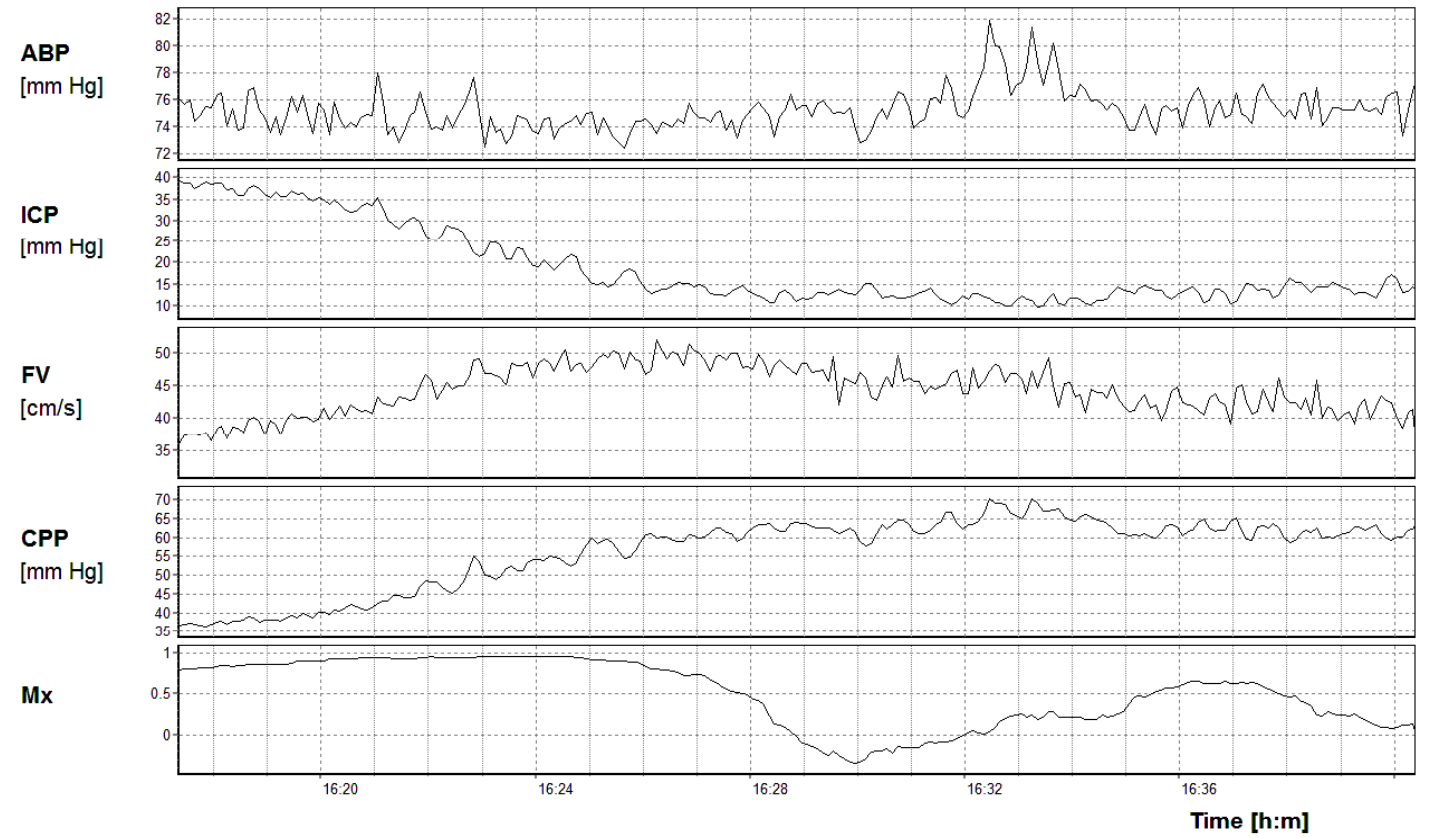

Fig. (2). Example of improving autoregulation (decrease in Mx) during a decrease in ICP after a period of intracranial hypertension.

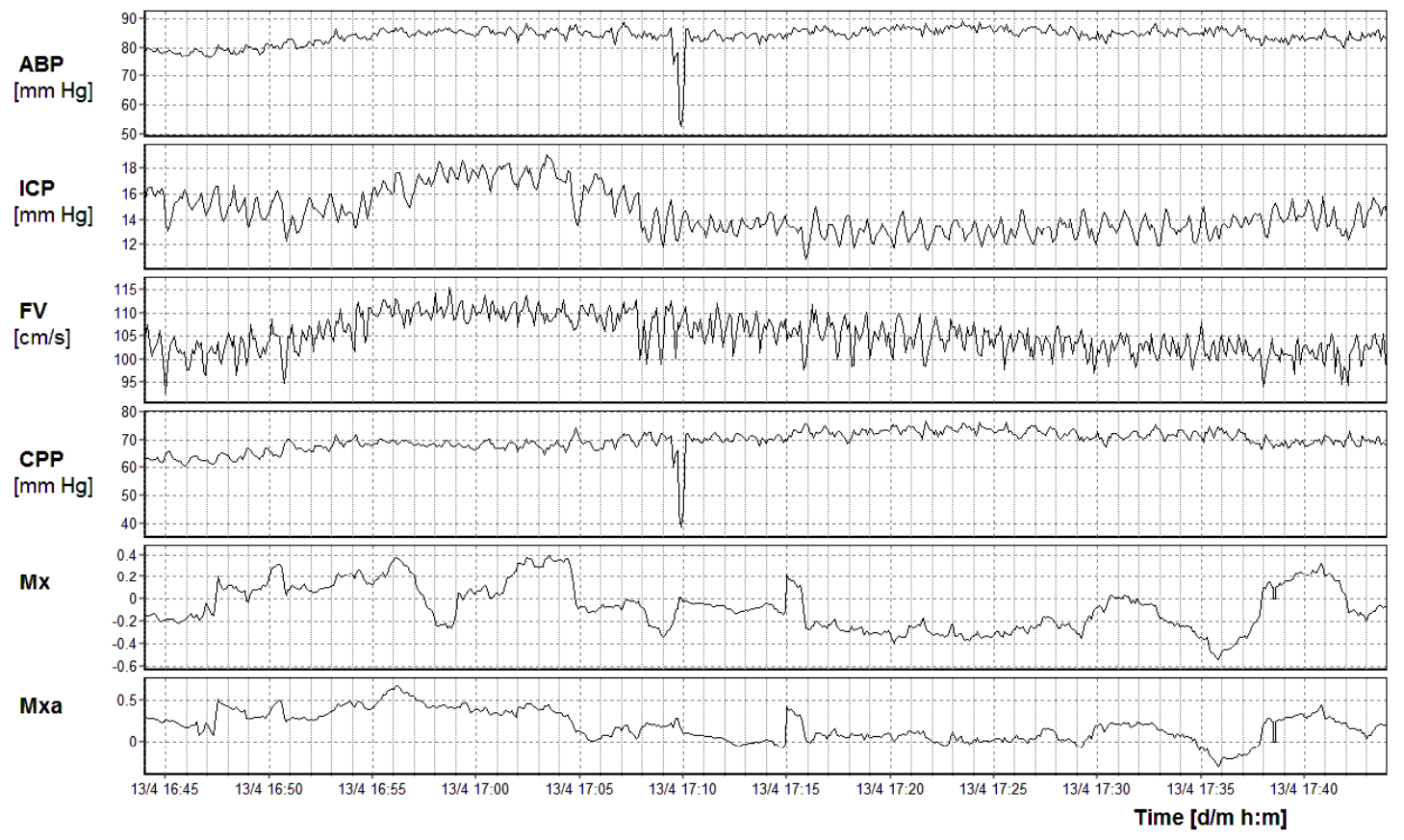

Fig. (3). Example of a good association between changes in autoregulation indices calculated using CPP (Mx) and ABP (Mxa). In those cases, where changes in CPP are mainly caused by changes by blood pressure, the association between Mx and Mxa is good (usually R $>0.7$, although Mxa is higher than Mx on average by 0.15). In cases where changes in CPP are mainly provoked by strong dynamics in ICP, Mxa and Mx may dramatically differ from each other.

transcranial Doppler (TCD), CBF may be approximated by cerebral blood flow velocity (CBFV) which is measured non-invasively at the bedside. Using TCD, different methods have been developed to monitor the cerebral autoregulation and critical closing pressure (CCP) by analyzing interactions between $\mathrm{CBFV}$ and $\mathrm{CPP}$ and between $\mathrm{CBFV}$ and $\mathrm{ABP}$.

\section{a. Cerebral Autoregulation and Critical Closing Pres- sure: Definitions and Validation}

Different methods have been defined to measure cerebral autoregulation using changes in $\mathrm{CBFV}$, in response to (spontaneous) changes in CPP and ABP. The index Mx is calculated in the time domain from the moving correlation 


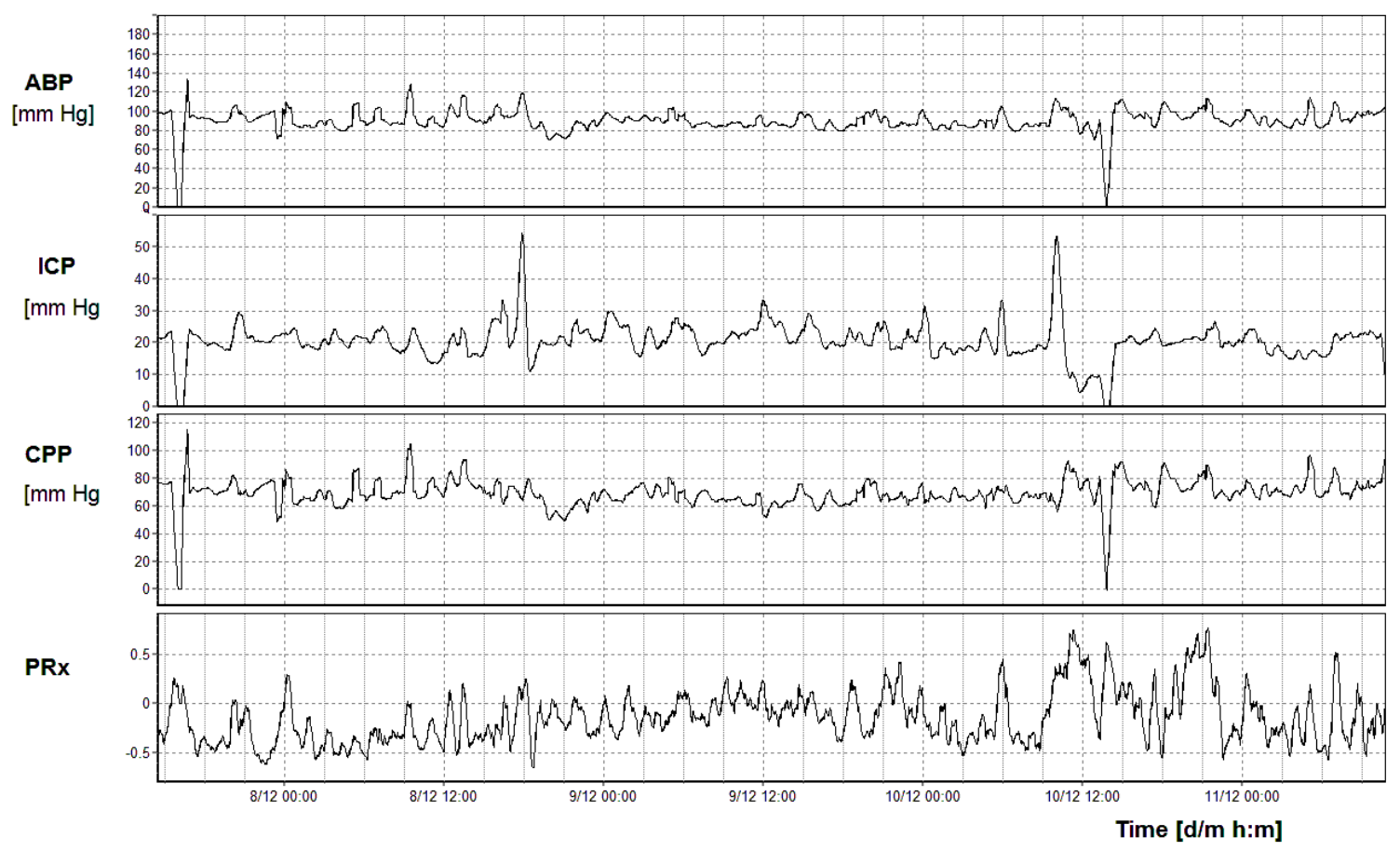

b

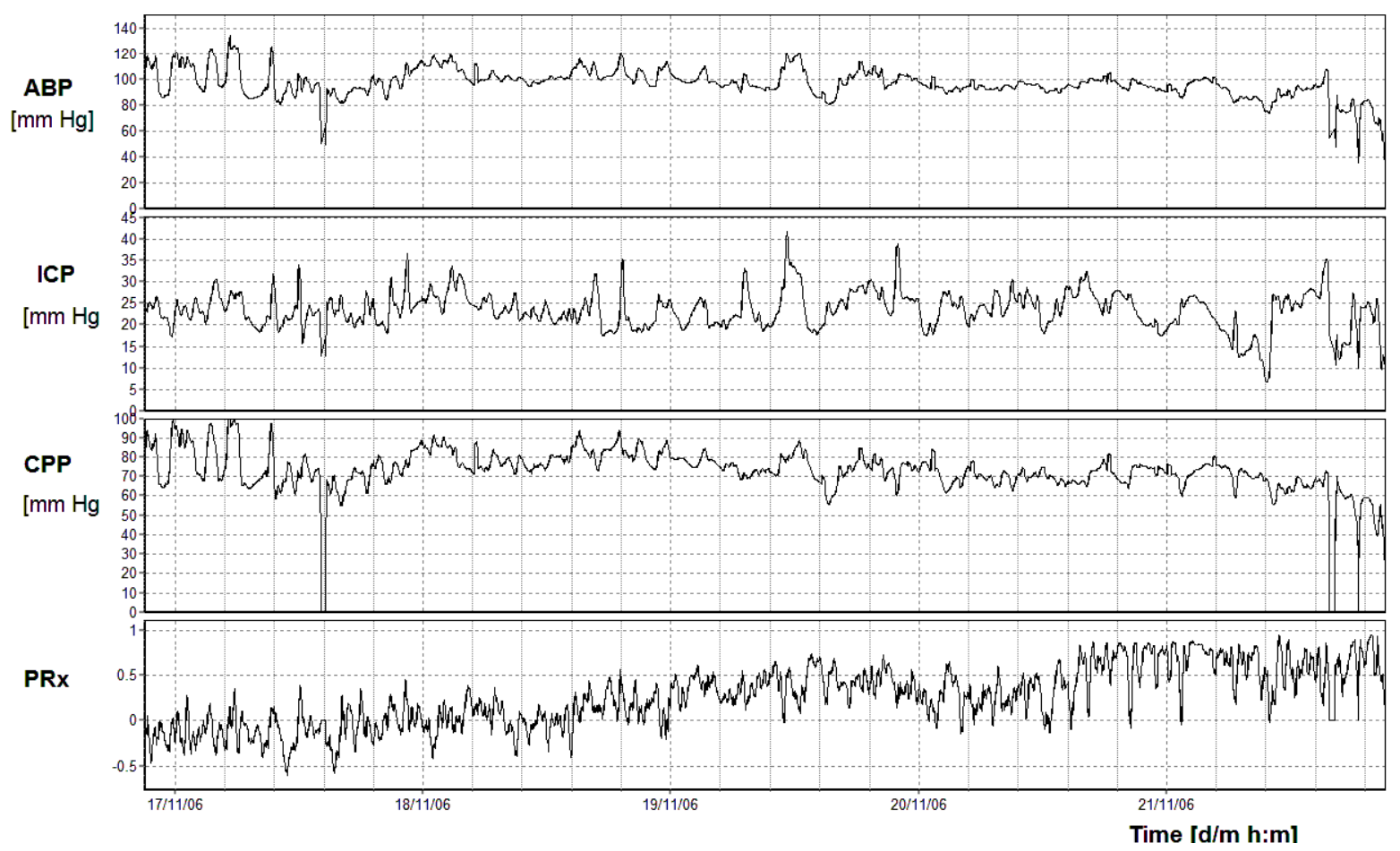

Fig. (4). Examples of monitoring of the cerebrovascular pressure reactivity index PRx in a patient after TBI. An obvious difference to the monitoring of Mx index is the period of monitoring:- PRx may be assessed continuously over the whole period of ICP/ABP monitoring (days or even weeks), while Mx, can only be monitored during the period of TCD monitoring.

a) Good outcome- despite a mean ICP of $20 \mathrm{~mm} \mathrm{Hg}$ and plateau waves reaching $60 \mathrm{~mm} \mathrm{Hg}$. Baseline PRx was negative (overall mean value around -0.2) with some elevations, e.g. 10/12 around 12:00 after a long plateau wave of ICP, lasting 30 minutes.

b) Patient with an unfavorable outcome, mean ICP was around $23 \mathrm{~mm} \mathrm{Hg}$. CPP was decreasing from 85 down to $60 \mathrm{~mm} \mathrm{Hg}$ and PRx- worsening from negative to positive values over 5 days of monitoring. 
between CBFV and CPP [7], whereas the index Mxa is defined as the moving correlation between CBFV and ABP [8]. Another method to measure dynamic autoregulation is the calculation of the autoregulation index (ARI), which is calculated by transfer function analysis to quantify the dynamic relationship between mean $\mathrm{ABP}$ and mean CBFV $[9,10]$. Phase shift between ABP and CBFV, and gain of the ABPCBFV transfer function have been also used [11].

The accuracy of Mx to measure cerebral autoregulation has been assessed by comparison with previously validated measures of static and dynamic autoregulation. In a study including 17 TBI patients, Mx was significantly correlated with the static rate of autoregulation (sROR), using increases in ABP induced by a norepinephrine infusion [12]. Additionally, $\mathrm{Mx}$ has been validated by comparison with other measures of dynamic cerebral autoregulation including the autoregulation index (ARI) [9], the leg-cuff test, and the transient hyperemic response [13].

Mxa is calculated as the moving correlation coefficient between CBFV and ABP instead of CPP, as used in the calculation of Mx. The ABP waveform may be measured directly from the radial or brachial artery or non-invasively using piezoelectric devices on radial or digital arteries [14]. Therefore, and on the contrary to Mx, Mxa may be calculated in a non-invasive way since it eliminates the need for invasive ICP measurements. Previous studies suggest that Mxa and Mx may be equivalent in assessing cerebral autoregulation. In a study including 37 TBI patients, a significant positive correlation between $\mathrm{CPP}$ and $\mathrm{CBFV}(\mathrm{Mx}>$ 0.3 ) was correlated with poor outcome. Similarly, in the same group of patients, a significant positive correlation between ABP and CBFV (Mxa > 0.45) was similarly a predictor of poor outcome, defined using the Glasgow Outcome Scale at discharge [8]. However, the measure of Mx seems more robust than the measure of Mxa, mainly due to the difficulties to measure $\mathrm{ABP}$ in a reliable manner due to frequent artifacts especially when ABP is recorded non-invasively. Therefore, although relatively similar in all patients, the differences between $\mathrm{Mx}$ and Mxa may be considerable in individual cases. Therefore, we would suggest that, when ICP is monitored, CPP rather than ABP should be used for the assessment of cerebral autoregulation (Figs. 1-3) [15].

Critical Closing Pressure (CCP) refers to the pressure at which cerebral vessels would collapse [16]. To estimate $\mathrm{CCP}$, different mathematical models have been described. In TBI patients, CCP has been used to detect moderate intracranial hypertension $[17,18]$. Additionally, based on a series of 119 patients [19], CCP was lower on the side of the contusion.

CBFV measured using TCD hase also been used to noninvasively estimate the ICP and CPP [20]. Newer algorithms to estimate ICP use the mean CBFV, the pulsatility index [21] or the combination of CBFV and ABP [20]. Different studies are currently being performed to determine the best algorithm to assess ICP optimize non-invasive ICP estimation using $\mathrm{CBFV}$ and $\mathrm{ABP}$ waveforms.

\section{b. Clinical Management and Prognostic Significance of Cerebral Autoregulation}

In clinical practice, $\mathrm{Mx}$ has demonstrated usefulness to monitor, grade, and reliably assess cerebral autoregulation without external stimulus, i.e. based on spontaneous fluctuations of CPP or ABP. Because Mx is a continuous index, a threshold separating normal and pathological values, if it exist, is probably individual and time-dependent. Nevertheless, using the average and daily value of day by day of $\mathrm{Mx}$, a cutoff of $\mathrm{Mx}>0.3$ seems to be adequate to determine

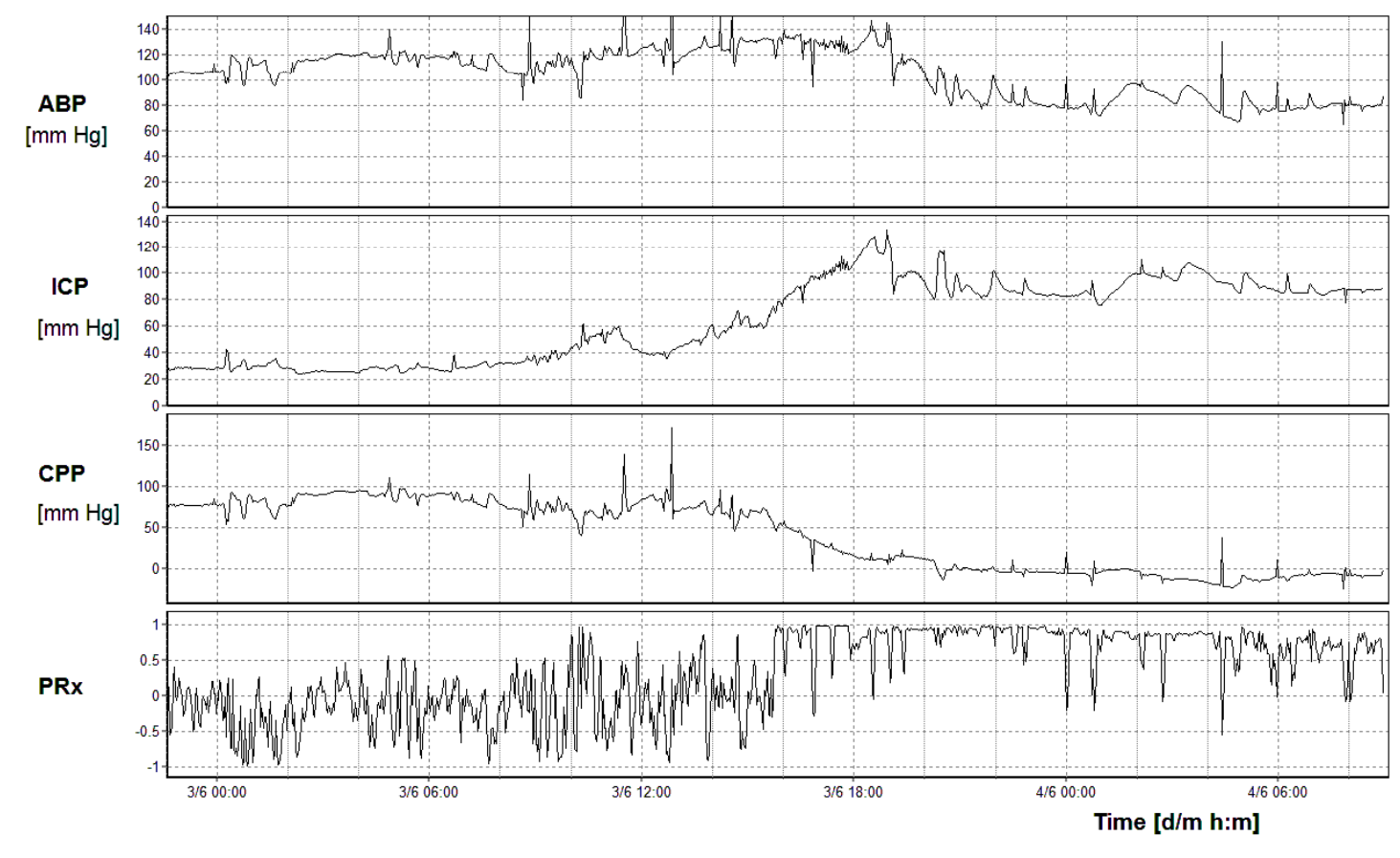

Fig. (5). Example of PRx increasing to $+1 \mathrm{~s}$ in a patient who developed refractory intracranial hypertension. Compare to Fig. (2)- both indices PRx and Mx react to intracranial hypertension, although they cannot be treated as a straightforward function of mean ICP. 
greater probability of achieving unfavorable outcome after TBI $[7,12]$. In a study of 187 patients, Mx was impaired not only when associated with a high ICP or low ABP, but also when CPP was abnormally elevated suggesting that Mx can be used to guide intensive care therapy when CPP-oriented protocols are used [22]. In ventilated patients, hyperventilation has shown to significantly impair cerebral autoregulation when measured using the auroregulation index (ARI) based on continuous measurement of ABP and CBFV waveforms [23]. In a prospective study of 122 patients suffering from severe TBI, the course of cerebral autoregulation has been studied during the first days after trauma. A progressive deterioration in the ARI was observed during the first 36 to 48 hours post injury, occurring despite an increase in CBFV. This evolution was not explained by changes in CPP or endtidal $\mathrm{CO}_{2}$. These results suggest that monitoring of cerebral autoregulation may be of value at least during the first week after TBI, and longer if the clinical condition requires it [24].

Several studies have demonstrated the prognostic role of Mx and Mxa after severe TBI. In a study including 82 patients, $\mathrm{Mx}$ above the threshold of 0.3 was predictive of poor outcome assed using the Glasgow outcome scale [7]. These results were confirmed in another study in which $\mathrm{Mx}$ above 0.3 was associated with poor outcome [12]. In another study based on 37 patients, in whom both Mxa and Mx were studied, both indices were correlated with poor outcome defined using the Glasgow outcome scale [8]. Because CBFV may be measured on the right and the left sides, an interhemispherical asymmetry in Mx has shown to be correlated with the side of contusion or brain expansion is some [25] but not all studies [26]. The side-to-side difference in Mx was a predictor of fatal outcome following TBI in one study [25]. Mx and Mxa are not the only indices assessing cerebral autoregulation associated with outcome. Impaired autoregulation measured by an abnormal ARI was strongly associated with bad outcome in TBI patients independent of the clinical pattern [27].

\section{INTRACRANIAL PRESSURE AND PRESSURE REACTIVITY}

In patients with severe TBI, evaluation of intracranial pressure (ICP) is recognized as a standard monitor the status of the cerebral pressure-volume regulation. This is particularly true when the patient is sedated limiting the possibilities of a complete clinical evaluation of neurological function. Active reduction of mean ICP is recommended when it exceeds a threshold of 20-25 $\mathrm{mmHg}$ [28]. However, recent studies have shown limited impact on outcome from treatment based on reduction of mICP only [29-31]. The development of software capable of real-time waveform analysis has generated various indices describing characteristics of the ICP waveform. Foremost among these indices is the pressure reactivity index $(\mathrm{PRx})$.

\section{a. Cerebrovascular Pressure Reactivity: Definition and Validation}

The pressure reactivity index (PRx) (hereafter pressure reactivity) is calculated as the moving correlation coefficient between slow waves in ABP and ICP [32]. In the case of the $\mathrm{PRx}, \mathrm{ICP}$ is used as surrogate measure of cerebral blood volume, measuring the ability of resistance arterioles to constrict and dilate in opposition to slow changes in ABP. In this respect, the PRx measures vascular reactivity, which is distinct from metrics of autoregulation. By contrast, the $\mathrm{Mx}$
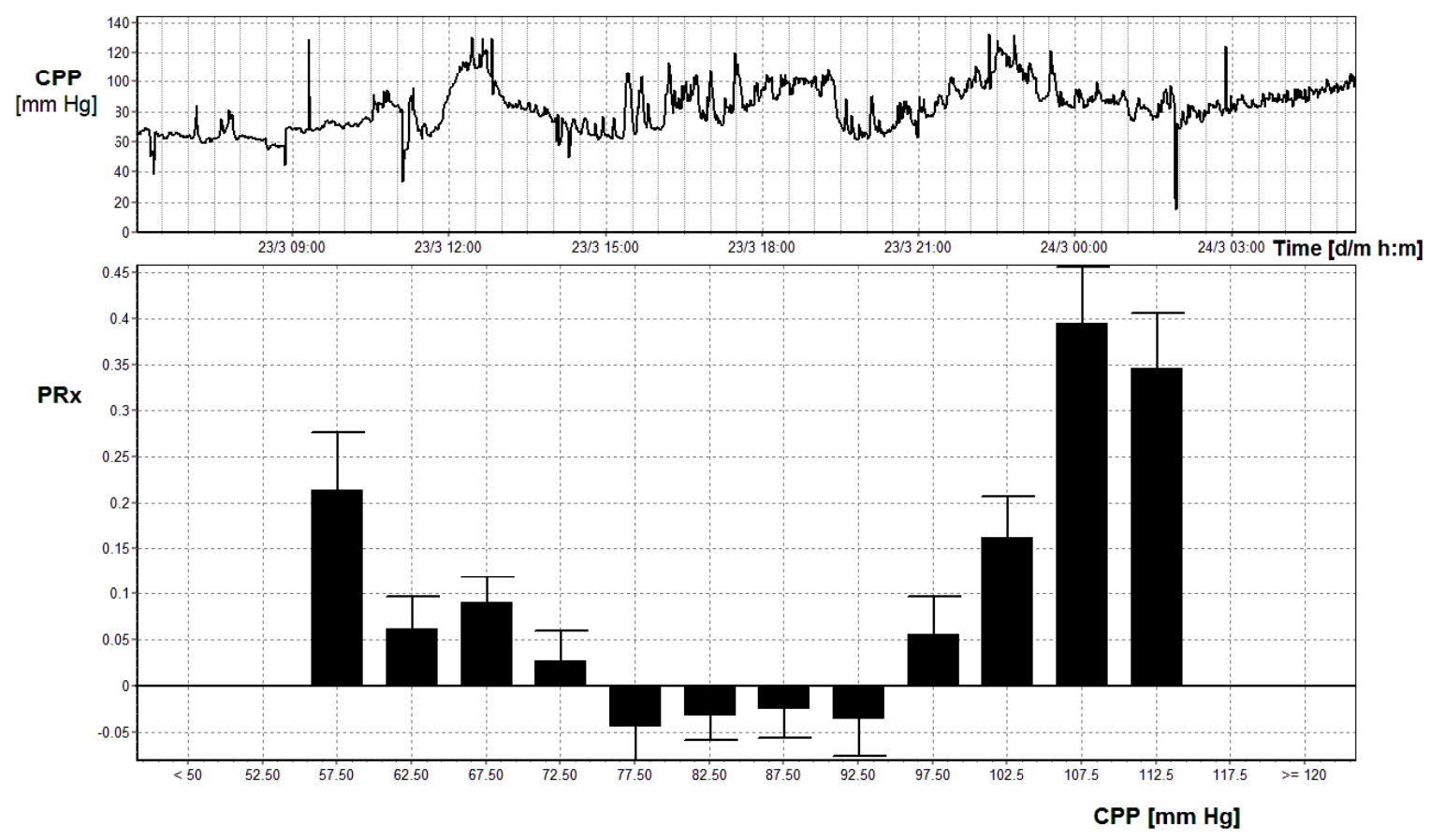

Fig. (6). Calculation of optimal CPP using the PRx index. Mean PRx is plotted against CPP over the period of the past few hours. In most of cases, this plot shows a U-shaped relationship. CPP associated with minimal and negative PRx is termed 'optimal CPP'- i.e. the individual level of CPP which is able to maintain the best achievable cerebrovascular pressure-reactivity. 
uses CBFV as a surrogate of cerebral blood flow to measure the ability of the cerebral vasculature to constrain flow. Thus, the $\mathrm{Mx}$ is a measure of pressure autoregulation, and the PRx is a measure of vascular reactivity, which is the mechanism of pressure autoregulation. The accuracy of PRx to reliably assess pressure reactivity with several validation techniques. In a study including 40 TBI patients, the relationship between PRx and CBF has been studied [33]. No significant correlation between $\mathrm{ABP}$ or CPP and CBFV (i.e. intact autoregulation) was reported in 19 patients with normal PRx but correlation between these variables was significant in 21 patients with impaired PRx. Additionally, PRx has been validated using PET scans. In a study including 22 patients, a low cerebral metabolic rate of oxygen $\left(\mathrm{CMRO}_{2}\right)$ was associated with a disturbed PRx and there was a significant correlation between impaired PRx and oxygen extraction fraction [34]. In another study, there was a significant correlation between PRx and the static rate of autoregulation measured with PET [35]. The PRx was also tested in a piglet model of hypotension and elevated ICP in which the lower limit of autoregulation was intentionally crossed and identified. Values of PRx became consistently elevated when the perfusion pressure was below this threshold. Further, the accuracy of the PRx was not affected by changes in intracranial compliance (Figs. 4, 5) [36].

\section{b. Cerebrovascular Pressure Reactivity: Clinical Appli- cations}

The role of PRx in the management of TBI patients and as a predictor of outcome has been scrutinized in different studies. In the clinical setting, recent guidelines recommend not allowing a CPP below $60 \mathrm{mmHg}$ [37]. Because this threshold may vary depending on the condition and treatment of the patient, the concept of "optimal cerebral perfusion pressure $\left(\mathrm{CPP}_{\mathrm{OPT}}\right)$ " was introduced using the $\mathrm{PRx}$ index.
In a retrospective study of $114 \mathrm{TBI}$ patients, the $\mathrm{CPP}_{\mathrm{OPT}}$ was defined as the CPP range associated with a minimal PRx value (indicating the most robust state of pressure reactivity). Patients who were managed with an average CPP close to the $\mathrm{CPP}_{\mathrm{OPT}}$ were more likely to have a favorable outcome than those whose mean CPP was further away from the $\mathrm{CPP}_{\text {OPT }}$ [38]. This suggests that PRx may be useful to determine, on an individual basis, the CPP range associated with the best outcome in TBI patients. 'Optimization' of CPP can be also understood as a searching for individual consensus between the 'Lund concept' which uees intentionally low CPP targets to minimize oedema formation, and classical "CPP -oriented therapy" [39], which sets higher CPP targets mitigate ischemic injury (Fig. 6) [40].

One of the major neurological complications requiring intensive treatment is raised ICP. Various strategies to reduce ICP are used in the clinical setting but their efficacy is still debated. Therefore, the response of PRx to different therapeutic strategies has been studied to determine the effect of treatment not only on ICP but also on pressure reactivity. Moderate hyperventilation was used historically to decrease ICP. When studies of intentional hypocapnea demonstrated a negative impact on outcome, the Brain Trauma Foundation recommended against the routine use of this practice which was subsequently abandoned by most centers [41-43]. In a study of 30 severe TBI patients, PRx was found to worsen with hyperventilation, although a large heterogeneity in the response to hypocapnia was found [44]. Hypothermia is increasingly used in the acute period of TBI to decrease ICP but its hemodynamic effects are largely unknown. A recent study, based on 24 patients with severe TBI, indicated that reactivity is not modified during hypothermia but did worsen during excessive rewarming indicating a significant derangement in cerebrovascular reactivity at this stage [45]. The effect of medications on PRx has been

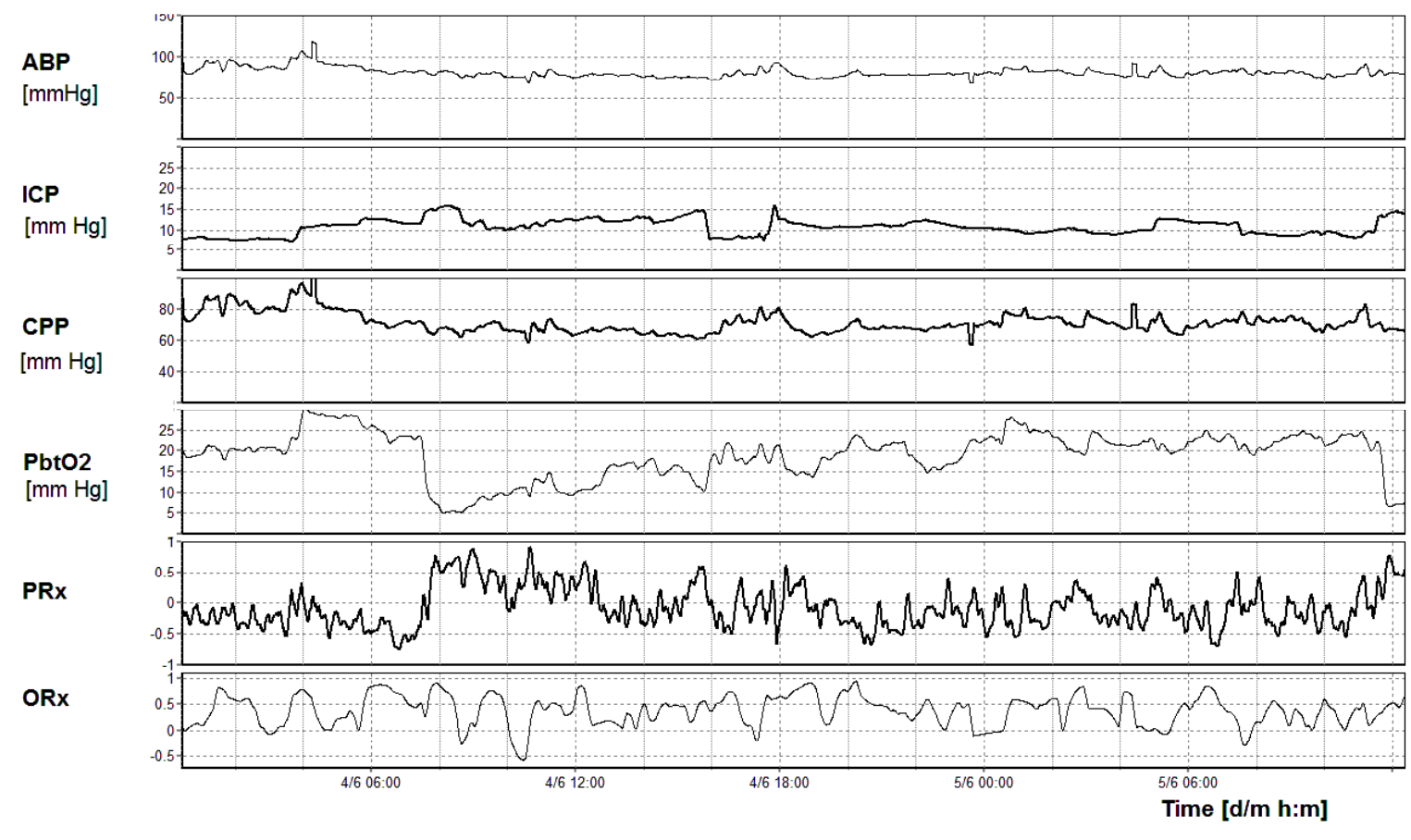

Fig. (7). Example of a poor correlation between changes in ORx and PRx. 
tested in various studies. Propofol is often used to sedate adult TBI patient. However, a study based on 10 patients showed a deterioration of pressure reactivity with rapid propofol infusion suggesting that large propofol doses may increase the injured brain's vulnerability to secondary insults [46]. On the contrary, barbiturate therapy for refractory intracranial hypertension after TBI improved PRx in a prospective observational study of 12 patients [47]. Another strategy to decrease refractory ICP is craniectomy, which is used in the most severe cases of refractory intracranial hypertension. Following decompressive craniectomy, pressure reactivity initially worsens, but improves in the later postoperative course [48], suggesting that craniectomy may contribute to the restoration of disturbed pressure-reactivity [49].

\section{c. Pressure Cerebrovascular Reactivity: Outcome}

The prognostic significance of PRx has been demonstrated in several studies. In one study of 98 TBI patients, a positive PRx correlated with high ICP, low admission GCS, and poor outcome at 6 months after injury [50]. Similarly, fatal outcome was associated with worse PRx, but also higher ICP and lower CPP in a more recent study [51]. A threshold PRx value of 0.3 has been proposed as value above 0.3 are associated with poor outcome. In a study of 193 patients, the mortality rose from $20 \%$ to $70 \%$ when the averaged PRx increased to above 0.3 [52]. More recently, based on a pooled dataset [53], a PRx above the threshold of 0.35 was associated with a high mortality (> 50\%). In a subset of patients with refractory intracranial hypertension, deterioration of the PRx was correlated with outcome, and was able to differentiate patients with good outcome, moderate disability, severe disability, and death. When multiple measured values of the PRx are plotted as a functionof CPP, a Ushaped curve often results, suggesting that too low and too high CPP was associated with a disturbance in pressure reactivity. The prognostic role of PRx has been further defined in a prospective study of 126 subjects with severe TBI using the Glasgow Outcome scale. This study showed that both mean ICP and PRx predict outcome when considered over the whole monitoring period. However, when looking at the first 24 hours, only PRx but not mean ICP was associated with outcome. The patients with disturbed pressure reactivity in the first 24 hours after injury had a significantly higher mortality rate than patients with intact pressure reactivity (29\% vs. $10 \%)$. These results suggest that the PRx may be an earlier predictor of outcome and therefore more useful in the "ultra-early" phase of TBI [54].

\section{BRAIN TISSUE OXYGENATION AND (PBTO $)_{2}$ AND OXYGEN REACTIVITY}

In TBI patients, brain tissue oxygenation $\left(\mathrm{PbtO}_{2}\right)$ is used as a predictive factor to detect brain ischemia and poor outcome. Different thresholds of ischemia have been evaluated and current recommendations suggest that the brain tissue with a $\mathrm{PbtO}_{2}$ value below $15 \mathrm{mmHg}$ may be considered high risk and values below $10 \mathrm{mmHg}$ are associated with irreversibly ischemia [55]. However, because $\mathrm{PbtO}_{2}$ depends on multiple variables including $\mathrm{CBF}, \mathrm{PaCO}_{2}, \mathrm{PaO}_{2}$ or $\mathrm{CPP}$, a treatment based on $\mathrm{PbtO}_{2}$ only has not been recommended. Using the same correlation method derived to calculate PRx, a new coefficient called the oxygen reactivity index (ORx) has been proposed. The Orx is the moving correlation between $\mathrm{PbtO}_{2}$ and CPP (Fig. 7) [56].

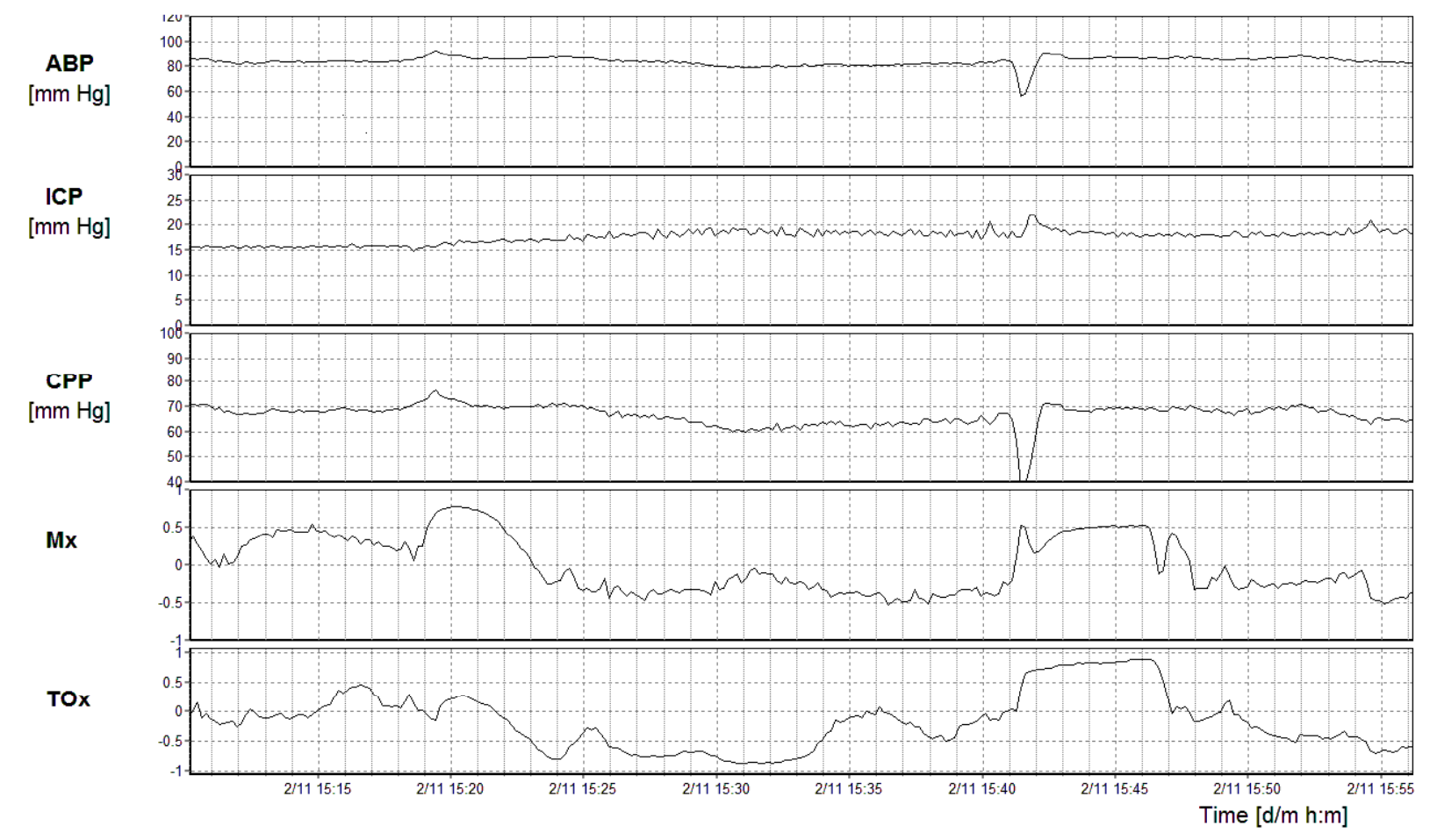

Fig. (8). Example of a good association between changes Tox and Mx index (R>0.8). This possiblysuggests that NIRS may be a good clinical tool for continuous monitoring of autoregulation of CBF. Application of NIRS is much easier than TCD probes (which should be continuously focused on MCA). 
The ORx has been validated by comparison with the static rate of regulation determined after pharmacologic blood pressure manipulations [57]. Limited studies have examined the ability of of $\mathrm{PbtO} 2$ and $\mathrm{ORx}$ to predict outcome. In a study of 40 subjects with TBI, the relationship between low $\mathrm{PbtO}_{2}$ and impaired PRx was significant in nonsurvivors but not in survivors [58]. A similar result was found in another study based on 27 patients suffering from severe TBI in which ORx and, to a lesser extent, $\mathrm{PbtO}_{2}$ correlated with $\mathrm{PRx}$ suggesting that that $\mathrm{Orx}$ and $\mathrm{PbtO}_{2}$ provide additional information about the status of cerebrovascular autoregulation after TBI. Furthermore, these data suggest that patients with impaired autoregulation are at increased risk for secondary cerebral hypoxia [59]. However, these promising results need yet to be confirmed as a recent retrospective study based on 32 patients did not show any correlation between Orx and PRx, limiting the role of ORx in the clinical practice pending further confirmation [60].

\section{E. OTHER MONITORING AND PERSPECTIVES}

In addition to $\mathrm{ABP}, \mathrm{CBFV}$, and ICP, different invasive and non-invasive tools to monitor patients with TBI are available or in developpement.

\section{a. Non-Invasive Techniques}

Near infrared spectroscopy (NIRS) is used to noninvasively measure oxy- and deoxy-hemoglobin. Many commercial NIRS-based monitors of the brain report oxyhemoglobin as a percentage of total hemoglobin measured in the reflected path of infrared light applied to the forehead. This measurement of whole-tissue oxyhemoglobin saturation is thought to represent the relative supply and uptake of oxygen from the blood. When hematocrit, arterial oxygen saturation, and cerebral metabolism are constant, as is the case for the periods used in time-domian autoregulation indices, this percent saturation is a function of cerebral blood flow. Recently, tissue oxyhemoglobin saturation obtained from NIRS monitors was compared to $\mathrm{ABP}$ to quantify cerebrovascular autoregulation $[61,62]$. Similar to previous indices, an index (Tox) was defined as the correlation coefficient between the tissue oxyhemoglobin saturation measured with NIRS and ABP. In a series of sepsis patients, this index Tox was highly correlated with Mxa, determined using CBFV and ABP. If confirmed in TBI patients, Tox may be a promising way to determine cerebral autoregulation non invasively without the inconvenient of transcranial Doppler [63]. This method was also shown to accurately detect the lower limit of autoregulation using a piglet model of hypotension [61]. Fig. (8) among other non-invasive techniques, different methods have focused on the cardiocirculatory system. This included monitoring of heart rate variability and cardiac output. In a recent study of TBI patients, low variability, and low baroreflex sensitivity, were linked to a higher mortality rate [64].

\section{b. Invasive Techniques}

With the use of intraparenchymatous probes, different variables may be recorded including brain metabolism using microdialysis, brain temperature and $\mathrm{CBF}$ using microprobes and thermodilution. However, because the information provided is not continuous, comparisons between these different techniques are difficult. Further development may help to provide a link between cerebral autoregulation and brain metabolism. Development of research in the domain of imaging may also help in this perspective. Only very few studies have investigated this relationship [65].

\section{CONCLUSION}

In the last two decades, multimodality monitoring has shown an impressive development. This development arises with the collaboration between researchers in the field of data processing and signal analysis and physicians treating TBI patients. Future collaborations have to be developed to share both knowledge and data. Today, it is not only monitoring which must be multimodal but also collaboration among researchers and clinicians from various institutions.

\section{ACKNOWLEDGEMENTS}

The authors are in debt to all the team participating in data collection: Mrs. Pippa Al-Rawi, Mrs. Helen Seley, Mrs. Carole Turner, Dr. Marcella Balestreri, Dr. Magda Hiler, Dr. Luzius Steiner, Dr. Eric Schmidt, Dr. Stefan Piechnik, Dr. Andreas Raabe, Mr. Eric Guazzo, Dr. David Menon, Dr. Arun Gupta, Dr. Basil Matta, Mr. Peter Kirkpatrick, Mr P.Hutchinson, Mr. Ivan Timofyeyev, Mr. Pwawanjit Minhas, Professor John D Pickard and all nursing and research staff of NCCU and Wolfson Brain Imaging Centre. The project was supported by the Swiss National Science Foundation (to EC) and the National institute of Health Research Biomedical Research Centre, Cambridge University Hospital Foundation Trust - Neurosciences Theme.

This work was supported by the Swiss National Science Foundation (PASSMP3-124262 to EC, and PBBSP3-125550 to $\mathrm{CZ}$ ), Bern, Switzerland.

\section{DISCLOSURE}

The software for brain monitoring ICM+ (www.neurosurg.cam.ac.uk/icmplus) is licensed by the University of Cambridge (Cambridge Enterprise). PS and MC have a financial interest in a part of the licensing fee. 
Tox = Tissue oxygenation index reactivity in response to a change in ABP

\section{REFERENCES}

[1] Maas AI, Stocchetti N, Bullock R. Moderate and severe traumatic brain injury in adults. Lancet Neurol 2008; 7: 728-41.

[2] Bhatia A, Gupta AK. Neuromonitoring in the intensive care unit. Ii. Cerebral oxygenation monitoring and microdialysis. Intensive Care Med 2007; 33: 1322-8.

[3] Bhatia A, Gupta AK. Neuromonitoring in the intensive care unit. I. Intracranial pressure and cerebral blood flow monitoring. Intensive Care Med 2007; 33: 1263-71.

[4] Guillaume J, Janny P. Continuous intracranial manometry; importance of the method and first results. Rev Neurol (Paris) 1951; 84: 131-42.

[5] Guendling K, Smielewski P, Czosnyka M, et al. Use of icm+ software for on-line analysis of intracranial and arterial pressures in head-injured patients. Acta Neurochir Suppl 2006; 96: 108-13.

[6] Smielewski P, Czosnyka M, Steiner L, Belestri M, Piechnik S, Pickard JD. Icm+: Software for on-line analysis of bedside monitoring data after severe head trauma. Acta Neurochir Suppl 2005; 95: 43-9.

[7] Czosnyka M, Smielewski P, Kirkpatrick P, Menon DK, Pickard JD. Monitoring of cerebral autoregulation in head-injured patients. Stroke 1996; 27 : 1829-34.

[8] Lang EW, Lagopoulos J, Griffith J, et al. Noninvasive cerebrovascular autoregulation assessment in traumatic brain injury: Validation and utility. J Neurotrauma 2003; 20: 69-75.

[9] Czosnyka M, Smielewski P, Lavinio A, Pickard JD, Panerai R. An assessment of dynamic autoregulation from spontaneous fluctuations of cerebral blood flow velocity: A comparison of two models, index of autoregulation and mean flow index. Anesth Analg 2008; 106: 234-9, table of contents.

[10] Tiecks FP, Lam AM, Aaslid R, Newell DW. Comparison of static and dynamic cerebral autoregulation measurements. Stroke 1995; 26: 1014-9.

[11] Muller M, Bianchi O, Erulku S, Stock C, Schwerdtfeger K. Brain lesion size and phase shift as an index of cerebral autoregulation in patients with severe head injury. Acta Neurochir (Wien) 2003; 145 : 643-7; discussion 647-8.

[12] Lang EW, Mehdorn HM, Dorsch NW, Czosnyka M. Continuous monitoring of cerebrovascular autoregulation: a validation study. J Neurol Neurosurg Psychiatry 2002; 72: 583-6.

[13] Smielewski P, Czosnyka M, Kirkpatrick P, Pickard JD. Evaluation of the transient hyperemic response test in head-injured patients. J Neurosurg 1997; 86: 773-8.

[14] Lavinio A, Schmidt EA, Haubrich C, Smielewski P, Pickard JD, Czosnyka M. Noninvasive evaluation of dynamic cerebrovascular autoregulation using finapres plethysmograph and transcranial doppler. Stroke 2007; 38: 402-4.

[15] Lewis PM, Smielewski P, Pickard JD, Czosnyka M. Dynamic cerebral autoregulation: Should intracranial pressure be taken into account? Acta Neurochir (Wien) 2007; 149: 549-55; discussion 555 .

[16] Burton AC. On the physical equilibrium of small blood vessels. Am J Physiol 1951; 164: 319-29.

[17] Czosnyka M, Smielewski P, Piechnik S, et al. Critical closing pressure in cerebrovascular circulation. J Neurol Neurosurg Psychiatry 1999; 66: 606-11.

[18] Edouard AR, Vanhille E, Le Moigno S, Benhamou D, Mazoit JX. Non-invasive assessment of cerebral perfusion pressure in brain injured patients with moderate intracranial hypertension. $\mathrm{Br} \mathrm{J}$ Anaesth 2005; 94: 216-21.

[19] Kumar A, Schmidt EA, Hiler M, Smielewski P, Pickard JD, Czosnyka M. Asymmetry of critical closing pressure following head injury. J Neurol Neurosurg Psychiatry 2005; 76: 1570-3.

[20] Schmidt EA, Czosnyka M, Gooskens I, et al. Preliminary experience of the estimation of cerebral perfusion pressure using transcranial doppler ultrasonography. J Neurol Neurosurg Psychiatry 2001; 70: 198-204.

[21] Voulgaris SG, Partheni M, Kaliora H, Haftouras N, Pessach IS, Polyzoidis KS. Early cerebral monitoring using the transcranial doppler pulsatility index in patients with severe brain trauma. Med Sci Monit 2005; 11: CR49-52.
[22] Czosnyka M, Smielewski P, Piechnik S, Steiner LA, Pickard JD. Cerebral autoregulation following head injury. J Neurosurg 2001; 95: 756-63.

[23] Newell DW, Weber JP, Watson R, Aaslid R, Winn HR. Effect of transient moderate hyperventilation on dynamic cerebral autoregulation after severe head injury. Neurosurgery 1996; 39: 35-43; discussion 43-34.

[24] Hlatky R, Furuya Y, Valadka AB, et al. Dynamic autoregulatory response after severe head injury. J Neurosurg 2002; 97: 1054-61.

[25] Schmidt EA, Czosnyka M, Smielewski P, Piechnik SK, Pickard JD. Asymmetry of cerebral autoregulation following head injury. Acta Neurochir Suppl 2002; 81: 133-4.

[26] Lang EW, Yip K, Griffith J, Lagopoulos J, Mudaliar Y, Dorsch NW. Hemispheric asymmetry and temporal profiles of cerebral pressure autoregulation in head injury. J Clin Neurosci 2003; 10: 670-3.

[27] Panerai RB, Kerins V, Fan L, Yeoman PM, Hope T, Evans DH. Association between dynamic cerebral autoregulation and mortality in severe head injury. Br J Neurosurg 2004; 18: 471-9.

[28] Bratton SL, Chestnut RM, Ghajar J, et al. Guidelines for the management of severe traumatic brain injury. Viii. Intracranial pressure thresholds. J Neurotrauma 2007; 24(Suppl 1): S55-8.

[29] Shafi S, Diaz-Arrastia R, Madden C, Gentilello L. Intracranial pressure monitoring in brain-injured patients is associated with worsening of survival. J Trauma 2008; 64: 335-40.

[30] Ratanalert S, Phuenpathom N, Saeheng S, Oearsakul T, Sripairojkul B, Hirunpat S. Icp threshold in cpp management of severe head injury patients. Surg Neurol 2004; 61: 429-34; discussion 434-25.

[31] Chambers IR, Treadwell L, Mendelow AD. Determination of threshold levels of cerebral perfusion pressure and intracranial pressure in severe head injury by using receiver-operating characteristic curves: An observational study in 291 patients. J Neurosurg 2001; 94: 412-6.

[32] Czosnyka M, Smielewski P, Kirkpatrick P, Laing RJ, Menon D, Pickard JD. Continuous assessment of the cerebral vasomotor reactivity in head injury. Neurosurgery 1997; 41: 11-7; discussion 17-9.

[33] Lang EW, Lagopoulos J, Griffith J, et al. Cerebral vasomotor reactivity testing in head injury: the link between pressure and flow. J Neurol Neurosurg Psychiatry 2003; 74: 1053-9.

[34] Steiner LA, Coles JP, Czosnyka M, et al. Cerebrovascular pressure reactivity is related to global cerebral oxygen metabolism after head injury. J Neurol Neurosurg Psychiatry 2003; 74: 765-70.

[35] Steiner LA, Coles JP, Johnston AJ, et al. Assessment of cerebrovascular autoregulation in head-injured patients: a validation study. Stroke 2003; 34: 2404-9.

[36] Brady KM, Lee JK, Kibler KK, Easley RB, Koehler RC, Shaffner DH. Continuous measurement of autoregulation by spontaneous fluctuations in cerebral perfusion pressure: comparison of 3 methods. Stroke 2008; 39: 2531-7.

[37] Bratton SL, Chestnut RM, Ghajar J, et al. Guidelines for the management of severe traumatic brain injury. Ix. Cerebral perfusion thresholds. J Neurotrauma 2007; 24(Suppl 1): S59-64.

[38] Steiner LA, Czosnyka M, Piechnik SK, et al. Continuous monitoring of cerebrovascular pressure reactivity allows determination of optimal cerebral perfusion pressure in patients with traumatic brain injury. Crit Care Med 2002; 30: 733-8.

[39] Howells T, Elf K, Jones PA, et al. Pressure reactivity as a guide in the treatment of cerebral perfusion pressure in patients with brain trauma. J Neurosurg 2005; 102: 311-7.

[40] Nordstrom CH. Physiological and biochemical principles underlying volume-targeted therapy--the "Lund concept". Neurocrit Care 2005; 2: 83-95.

[41] Marion DW, Spiegel TP. Changes in the management of severe traumatic brain injury: 1991-1997. Crit Care Med 2000; 28: 16-8.

[42] Bratton SL, Chestnut RM, Ghajar J, et al. Guidelines for the management of severe traumatic brain injury. Xiv. Hyperventilation. J Neurotrauma 2007; 24(Suppl 1): S87-90.

[43] Steiner LA, Balestreri M, Johnston AJ, et al. Sustained moderate reductions in arterial co 2 after brain trauma time-course of cerebral blood flow velocity and intracranial pressure. Intensive Care Med 2004; 30: 2180-7.

[44] Steiner LA, Balestreri M, Johnston AJ, et al. Effects of moderate hyperventilation on cerebrovascular pressure-reactivity after head injury. Acta Neurochir Suppl 2005; 95: 17-20. 
[45] Lavinio A, Timofeev I, Nortje J, et al. Cerebrovascular reactivity during hypothermia and rewarming. Br J Anaesth 2007; 99: 23744.

[46] Steiner LA, Johnston AJ, Chatfield DA, et al. The effects of largedose propofol on cerebrovascular pressure autoregulation in headinjured patients. Anesth Analg 2003; 97: 572-6, table of contents.

[47] Thorat JD, Wang EC, Lee KK, Seow WT, Ng I. Barbiturate therapy for patients with refractory intracranial hypertension following severe traumatic brain injury: Its effects on tissue oxygenation, brain temperature and autoregulation. J Clin Neurosci 2008; 15: 143-8.

[48] Timofeev I, Czosnyka M, Nortje J, et al. Effect of decompressive craniectomy on intracranial pressure and cerebrospinal compensation following traumatic brain injury. J Neurosurg 2008; 108: 6673.

[49] Wang EC, Ang BT, Wong J, Lim J, Ng I. Characterization of cerebrovascular reactivity after craniectomy for acute brain injury. Br J Neurosurg 2006; 20: 24-30.

[50] Czosnyka M, Smielewski P, Kirkpatrick P, Piechnik S, Laing R, Pickard JD. Continuous monitoring of cerebrovascular pressurereactivity in head injury. Acta Neurochir Suppl 1998; 71: 74-7.

[51] Czosnyka M, Hutchinson PJ, Balestreri M, Hiler M, Smielewski P, Pickard JD. Monitoring and interpretation of intracranial pressure after head injury. Acta Neurochir Suppl 2006; 96: 114-8.

[52] Balestreri M, Czosnyka M, Steiner LA, et al. Association between outcome, cerebral pressure reactivity and slow icp waves following head injury. Acta Neurochir Suppl 2005; 95: 25-8.

[53] Zweifel C, Lavinio A, Steiner LA, et al. Continuous monitoring of cerebrovascular pressure reactivity in patients with head injury. Neurosurg Focus 2008; 25: E2.

[54] Hiler M, Czosnyka M, Hutchinson P, et al. Predictive value of initial computerized tomography scan, intracranial pressure, and state of autoregulation in patients with traumatic brain injury. J Neurosurg 2006; 104: 731-7.
[55] Bratton SL, Chestnut RM, Ghajar J, et al. Guidelines for the management of severe traumatic brain injury. X. Brain oxygen monitoring and thresholds. J Neurotrauma 2007; 24(Suppl 1): S65-70.

[56] Soehle M, Jaeger M, Meixensberger J. Online assessment of brain tissue oxygen autoregulation in traumatic brain injury and subarachnoid hemorrhage. Neurol Res 2003; 25: 411-7.

[57] Lang EW, Czosnyka M, Mehdorn HM. Tissue oxygen reactivity and cerebral autoregulation after severe traumatic brain injury. Crit Care Med 2003; 31: 267-71.

[58] Ang BT, Wong J, Lee KK, Wang E, Ng I. Temporal changes in cerebral tissue oxygenation with cerebrovascular pressure reactivity in severe traumatic brain injury. J Neurol Neurosurg Psychiatry 2007; 78: 298-302.

[59] Jaeger M, Schuhmann MU, Soehle M, Meixensberger J. Continuous assessment of cerebrovascular autoregulation after traumatic brain injury using brain tissue oxygen pressure reactivity. Crit Care Med 2006; 34: 1783-8.

[60] Radolovich DK, Czosnyka M, Timofeev I, et al. Reactivity of brain tissue oxygen to change in cerebral perfusion pressure in head injured patients. Neurocrit Care 2009; 64: 494-501.

[61] Brady KM, Lee JK, Kibler KK, et al. Continuous time-domain analysis of cerebrovascular autoregulation using near-infrared spectroscopy. Stroke 2007; 38: 2818-25.

[62] Lee JK, Kibler KK, Benni PB, et al. Cerebrovascular reactivity measured by near-infrared spectroscopy. Stroke. 2009; 40: 1820-6.

[63] Steiner LA, Pfister D, Strebel SP, Radolovich D, Smielewski P, Czosnyka M. Near-infrared spectroscopy can monitor dynamic cerebral autoregulation in adults. Neurocrit Care 2009; 10: 122-8.

[64] Papaioannou V, Giannakou M, Maglaveras N, Sofianos E, Giala M. Investigation of heart rate and blood pressure variability, baroreflex sensitivity, and approximate entropy in acute brain injury patients. J Crit Care 2008; 23: 380-6.

[65] Valadka AB, Hlatky R, Furuya Y, Robertson CS. Brain tissue $\mathrm{PO}_{2}$ : Correlation with cerebral blood flow. Acta Neurochir Suppl 2002; 81: 299-301.

(c) Carrera et al.; Licensee Bentham Open.

This is an open access article licensed under the terms of the Creative Commons Attribution Non-Commercial License (http://creativecommons.org/licenses/by-nc/3.0/) which permits unrestricted, non-commercial use, distribution and reproduction in any medium, provided the work is properly cited. 